\begin{tabular}{|c|l|}
\hline Title & A nomal ous surface impedance in a normal-metal /superconductor junction with a spin-active interface \\
\hline Author(s) & A sano, Y asuhiro; Ozaki, Masahiro; Habe, Tetsuro; Golubov, A lex ander A.; T anaka, Y ukio \\
\hline Citation & $\begin{array}{l}\text { Physical Review B, 86(2), 024510 } \\
\text { https://doi.org/10.1103/PhysRevB.86.024510 }\end{array}$ \\
\hline Issue Date & 2012-07-01 \\
\hline Doc URL & http://hdl.handle.net/2115/49739 \\
\hline Rights & O2012A A merican Physical Society \\
\hline Type & article \\
\hline File Information & PRB86-2_024510.pdf \\
\hline
\end{tabular}

Instructions for use 


\title{
Anomalous surface impedance in a normal-metal/superconductor junction with a spin-active interface
}

\author{
Yasuhiro Asano \\ Department of Applied Physics and Center for Topological Science \& Technology, Hokkaido University, Sapporo 060-8628, Japan \\ Masahiro Ozaki and Tetsuro Habe \\ Department of Applied Physics, Hokkaido University, Sapporo 060-8628, Japan
}

\author{
Alexander A. Golubov \\ Faculty of Science and Technology and MESA + Institute of Nanotechnology, University of Twente, 7500 AE, Enschede, The Netherlands \\ Yukio Tanaka \\ Department of Applied Physics, Nagoya University, Nagoya 464-8603, Japan
}

(Received 18 April 2012; published 11 July 2012)

\begin{abstract}
We discuss the surface impedance $(Z=R-i X)$ of a normal-metal/superconductor proximity structure taking into account the spin-dependent potential at the junction interface. Because of the spin-mixing transport at the interface, odd-frequency spin-triplet $s$-wave Cooper pairs penetrate into the normal metal and cause the anomalous response to electromagnetic fields. At low temperature, the local impedance at a surface of the normal metal shows the nonmonotonic temperature dependence and the anomalous relation $R>X$. We also discuss a possibility of observing such anomalous impedance in experiments.
\end{abstract}

DOI: 10.1103/PhysRevB.86.024510

PACS number(s): 74.45.+c, 74.50.+r, 74.25.F-, 74.70.-b

\section{INTRODUCTION}

Physics of odd-frequency Cooper pairs ${ }^{1}$ has been a hot issue since a theoretical paper pointed out the existence of odd-frequency pairs in realistic proximity structures. ${ }^{2}$ There are mainly two ways to create the odd-frequency Cooper pairs in proximity structures. At first, spin mixing due to spin-dependent potential should generate odd-frequency pairs. The authors of Ref. 2 considered a ferromagnet/metallicsuperconductor junction, where the direction of magnetic moment near the interface is spatially inhomogeneous. The spin-flip scattering in such a magnetically inhomogeneous segment produces the odd-frequency spin-triplet $s$-wave Cooper pairs in the ferromagnet. This prediction has promoted a number of theoretical studies. ${ }^{3-11}$ Manifestations of triplet pairs were recently observed experimentally as a long-range Josephson coupling across ferromagnets. ${ }^{12-15}$ Alternatively, the odd-frequency pair was suggested in proximity structures involving a normal metal attached to an odd-parity spin-triplet superconductor that belongs to the conventional even-frequency symmetry class. The parity mixing due to inhomogeneity produces the odd-frequency pairs even in this case. ${ }^{16}$ The unusual properties of spin-triplet superconducting junctions due to odd-frequency pairs ${ }^{17-22}$ were predicted theoretically. Unfortunately, however, we have never had clear scientific evidences of odd-frequency pairs in experiments. This is because physical values focused in experiments have only indirect information of the frequency symmetry.

In a previous paper, ${ }^{23}$ we showed that the surface impedance directly reflects the frequency symmetry of Cooper pair. Surface impedance $Z=R-i X$ represents the dynamic response of Cooper pairs to low-frequency electromagnetic field. ${ }^{24,25}$ The surface resistance $R$ corresponds to resistance due to normal electrons. The reactance $X$ represents power loss of electromagnetic field due to Cooper pairs. In conventional even-frequency superconductors, the positive amplitude of the
Cooper-pair density guarantees a robust relation $R \ll X$ at low temperatures and at low frequencies. The validity of the relation $R<X$, however, is questionable for odd-frequency Cooper pairs because the odd-frequency symmetry and negative pair density are inseparable from each other according to the standard theory of superconductivity. ${ }^{26}$ We have considered a normal-metal/superconductor (NS) junction where superconductor belongs to spin-triplet odd-parity symmetry. On the basis of the quasiclassical Green's function method, we have theoretically shown that the odd-frequency Cooper pairs in the normal metal lead to the unusual relationship $R>X$. Therefore, observing the relation $R>X$ in experiments can be a very clear and direct evidence which suggests the existence of odd-frequency Cooper pairs. Although the detection of such unusual relation is possible these days, the fabrication of a well-characterized NS junction using chiral $p$-wave spin-triplet superconductor $\mathrm{Sr}_{2} \mathrm{RuO}_{4}$ (Ref. 27) is not an easy task. Thus, we need to discuss a possibility for observing the unusual relationship $R>X$ in other accessible proximity structures.

In this paper, we discuss the surface impedance in the NS junction consisting of a metallic superconductor where pairing symmetry belongs to spin-singlet $s$ wave. At the junction interface, we introduce a thin ferromagnetic layer which produces the odd-frequency spin-triplet $s$-wave Cooper pairs in the normal metal. The local complex conductivity is calculated based on the linear response theory using the quasiclassical Green's function method. We will conclude that the local impedance in the normal metal shows the unusual relation $R>X$ when the odd-frequency pairs are dominant in the normal metal. We also discuss a possibility to detect the relation in experiments.

This paper is organized as follows. In Sec. II, we explain the theoretical model of a NS junction and the formula for complex conductivity. The calculated results of impedance in 


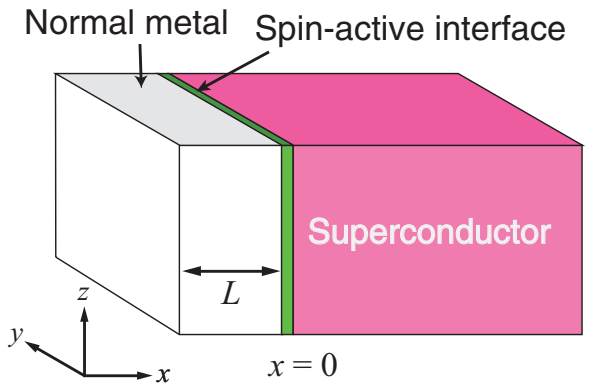

FIG. 1. (Color online) The schematic picture of a normalmetal/superconductor junction under consideration.

NS junctions are shown in Sec. III. The conclusion is given in Sec. IV.

\section{MODEL AND METHOD}

Let us consider a bilayer of a superconductor and a thin normal-metal film as shown in Fig. 1, where $L$ is the thickness of the normal metal. At the interface between the normal metal and the superconductor, we insert a magnetic thin film. This theoretical junction corresponds to the experimental junction, ${ }^{28}$ consisting of a superconductor and a ferromagnetic semiconductor GdN. The exchange potential at GdN causes spin-dependent transport through the ferromagnetic film. To calculate the complex conductivity in the normal metal, we first solve the quasiclassical Usadel equation ${ }^{29}$ in the standard $\theta$ parametrization:

$$
\hbar D \frac{d^{2} \theta_{\nu}(x, \epsilon)}{d x^{2}}+2 i \epsilon \sin \theta_{\nu}(x, \epsilon)=0,
$$

where $D$ is the diffusion constant of the normal metal and $\epsilon$ is the quasiparticle energy measured from the Fermi level. The subscript $v= \pm 1$ describes two Nambu spaces: $v=1$ indicates the subspace for electron spin up and hole spin down, and $v=-1$ indicates that for electron spin down and hole spin up.

The spin-dependent potential at the ferromagnetic semiconductor/insulator is easily considered through the boundary condition for wave function when we solve the Bogoliubov-de Gennes equation. ${ }^{30}$ The effects of spin-dependent scatterings on the Green's function are taken into account in the boundary condition at the NS interface: $:^{7,31-34}$

$$
\left.\gamma_{B} \frac{d \theta_{v}}{d x}\right|_{x=0}=\sin \left(\theta_{v}-\theta_{S}\right)+i v \frac{G_{\phi}}{G_{T}} \sin \theta_{\nu},
$$

where $\gamma_{B}=L \frac{R_{b}}{R_{d}}$ is an interface parameter with $R_{d}$ and $R_{b}$ being the resistance of the normal metal and that of the NS interface, respectively. The Green's function in the superconductor is described by

$$
\begin{gathered}
g_{S}=\cos \theta_{S}=\frac{\epsilon}{\sqrt{(\epsilon+i \lambda)^{2}-\Delta}}, \\
f_{S}=\sin \theta_{S}=\frac{i \Delta}{\sqrt{(\epsilon+i \lambda)^{2}-\Delta}},
\end{gathered}
$$

where $\Delta$ is the amplitude of pair potential in the bulk superconductor and $\lambda$ is a small parameter providing the retarded Green's function. The second term in Eq. (2) describes the spin-mixing effect at the junction interface. $G_{T}$ represents the spin-independent tunneling conductance of the junction interface, whereas $G_{\phi}$ is the spin-mixing conductance. ${ }^{35}$ At the outer surface of the normal metal, we require

$$
\left.\frac{\partial \theta(x, \epsilon)}{\partial x}\right|_{x=-L}=0 .
$$

The normal and anomalous retarded Green's functions are obtained as

$$
g_{v}(x, \epsilon)=\cos \theta_{v}(x, \epsilon), \quad f_{v}(x, \epsilon)=\sin \theta_{\nu}(x, \epsilon),
$$

respectively.

Having found the Green's functions, we can calculate the local complex conductivity that describes the response of the sample to the electromagnetic field. The local complex conductivity $\sigma_{\mathrm{N}}(x, \omega)=\sigma_{1}+i \sigma_{2}$ at frequency $\omega$ is determined by the general expression ${ }^{37}$

$$
\begin{gathered}
\frac{\sigma_{1}(x, \omega)}{\sigma_{0}}=\frac{1}{4 \hbar \omega} \int_{-\infty}^{\infty} d \epsilon[J(\epsilon+\hbar \omega)-J(\epsilon)] K_{1}, \\
\frac{\sigma_{2}(x, \omega)}{\sigma_{0}}=\frac{1}{4 \hbar \omega} \int_{-\infty}^{\infty} d \epsilon\left[J(\epsilon+\hbar \omega) K_{2}+J(\epsilon) K_{3}\right], \\
K_{1}=\sum_{\nu} f_{v, I}(\epsilon) f_{v, I}(\epsilon+\hbar \omega)+g_{v, R}(\epsilon) g_{v, R}(\epsilon+\hbar \omega), \\
K_{2}=\sum_{v} f_{v, R}(\epsilon) f_{v, I}(\epsilon+\hbar \omega)-g_{v, I}(\epsilon) g_{v, R}(\epsilon+\hbar \omega), \\
K_{3}=\sum_{v} f_{v, R}(\epsilon+\hbar \omega) f_{v, I}(\epsilon)-g_{v, I}(\epsilon+\hbar \omega) g_{v, R}(\epsilon), \\
\text { with } J(\epsilon)=\tanh \left(\epsilon / 2 k_{B} T\right) \text { and } \\
g_{v, R}(\epsilon)=\operatorname{Re}\left[g_{v}(x, \epsilon)\right], \quad g_{v, I}(\epsilon)=\operatorname{Im}\left[g_{v}(x, \epsilon)\right], \\
f_{v, R}(\epsilon)=\operatorname{Re}\left[f_{v}(x, \epsilon)\right], \quad f_{v, I}(\epsilon)=\operatorname{Im}\left[f_{v}(x, \epsilon)\right] .
\end{gathered}
$$

The local impedance in the normal metal is calculated from the complex conductivity as

$$
Z_{\mathrm{N}}(x, \omega)=R_{\mathrm{N}}-i X_{\mathrm{N}}=(1-i) \sqrt{\frac{\hbar \omega}{\Delta_{0}} \frac{\sigma_{0}}{\sigma_{\mathrm{N}}(x, \omega)}} Z_{0},
$$

where $Z_{0} \equiv \sqrt{2 \pi \Delta_{0} / \sigma_{0} c^{2} \hbar}, \Delta_{0}$ is the amplitude of pair potential at $T=0$, and $\sigma_{0}$ is the Drude conductivity in the normal metal. The derivation of the local conductivity is given in Appendix A. In this paper, we describe the dependence of $\Delta$ on temperature by the BCS theory. In particular, we focus on the local impedance at the surface of the normal metal defined by

$$
Z_{L}=R_{L}-i X_{L} \equiv Z_{\mathrm{N}}(-L, \omega) .
$$

Such local impedance is an accessible observable these days. ${ }^{36}$ Usual experiments measure the impedance of the whole NS structure, which is calculated as

$$
Z_{\mathrm{NS}}=R_{\mathrm{NS}}-i X_{\mathrm{NS}}=\bar{Z}_{\mathrm{N}} \frac{Z_{\mathrm{S}} \cos \bar{k}_{n} L-i \bar{Z}_{\mathrm{N}} \sin \bar{k}_{n} L}{\bar{Z}_{\mathrm{N}} \cos \bar{k}_{n} L-i Z_{\mathrm{S}} \sin \bar{k}_{n} L},
$$

where $Z_{\mathrm{S}}$ is the impedance of superconductor which is obtained by substituting the Green's function of superconductor in Eqs. (3) and (4) into Eqs. (7)-(13). The derivation of Eq. (16) is given in Appendix B. In this paper, $L$ is chosen to be comparable to $\xi_{T_{c}}=\sqrt{\hbar D / 2 \pi T_{C}}$ with $T_{C}$ the superconducting transition temperature. In such junctions, the conductivity is 
almost independent of $x$ in the normal metal. Therefore, it is possible to define spatially averaged values of the conductivity, the impedance, and the wave number of electromagnetic field as follows:

$$
\begin{gathered}
\bar{\sigma}_{\mathrm{N}}=\int_{-L}^{0} d x \sigma_{\mathrm{N}}(x) / L, \\
\bar{Z}_{\mathrm{N}}=\bar{R}_{\mathrm{N}}-i \bar{X}_{\mathrm{N}}=-i \sqrt{4 \pi i \omega /\left(c^{2} \bar{\sigma}_{\mathrm{N}}\right)}, \\
\bar{k}_{n}=\sqrt{i 4 \pi \omega \bar{\sigma}_{\mathrm{N}} / c^{2}} .
\end{gathered}
$$

To understand the relation between the frequency symmetry of a Cooper pair and the sign of the imaginary part of complex conductivity $\sigma_{2}$, we analyze the spectral pair density defined by

$$
\begin{gathered}
K_{s}(\epsilon)=\sum_{\nu= \pm 1} K_{s}(\nu, \epsilon), \\
K_{s}(\nu, \epsilon)=f_{v, R}(\epsilon) f_{v, I}(\epsilon)-g_{v, R}(\epsilon) g_{v, I}(\epsilon), \\
=2 f_{v, R}(\epsilon) f_{v, I}(\epsilon)=\operatorname{Im} f_{v}^{2}(\epsilon),
\end{gathered}
$$

which appears in the integrand of $\sigma_{2}$ in Eq. (8) at very small $\omega$. We used the normalization condition $g_{v}^{2}(\epsilon)+f_{v}^{2}(\epsilon)=1$. The spectral pair density contains full information about the symmetry of $f(\epsilon)$ and, therefore, the frequency symmetry of Cooper pairs. At $T=0$, the Cooper-pair density in the normal metal is

$$
n_{s}=\int_{-\infty}^{\infty} d \epsilon J(\epsilon) K_{s}(\epsilon) .
$$

Since $K_{s}$ is an odd function of $\epsilon$ according to its definition and $J(\epsilon)$ is also an odd step function of $\epsilon$, the pair density becomes

$$
n_{s}=2 \int_{0}^{\infty} d \epsilon K_{s}(\epsilon) .
$$

Finally, the local density of states is given by

$$
\begin{aligned}
& N(\epsilon, x)=\sum_{\nu} N(\epsilon, x, \nu), \\
& N(\epsilon, x, \nu)=\operatorname{Re}\left[g_{v}(\epsilon, x)\right],
\end{aligned}
$$

which is normalized to the normal density of states at the Fermi level.

\section{RESULTS}

The theory includes several independent parameters discussed as follows. Throughout this paper, we fix the thickness of a normal metal $L$ at $\xi_{T_{c}}$. The spatial dependence of the Green's function in the normal metal becomes weak in this choice. In numerical simulation, we do not discuss details of the averaged impedance $\bar{Z}_{\mathrm{N}}$ in Eq. (18) because we have confirmed that $Z_{L} \approx \bar{Z}_{\mathrm{N}}$. The second parameter $R_{d} / R_{b}$ tunes the degree of the proximity effect in a normal metal. The larger $R_{d} / R_{b}$ gives the stronger proximity effect. The third one is $G_{\phi} / G_{T}$, which represents the strength of the spin-dependent potential at the NS interface. The fourth one is the frequency of electromagnetic field $\omega$ which should be smaller than $\Delta_{0} / \hbar$ to obtain information about Cooper pairs. Finally, we fix the small imaginary part in energy as $\lambda=0.001 \Delta_{0}$, which does not affect following conclusions.

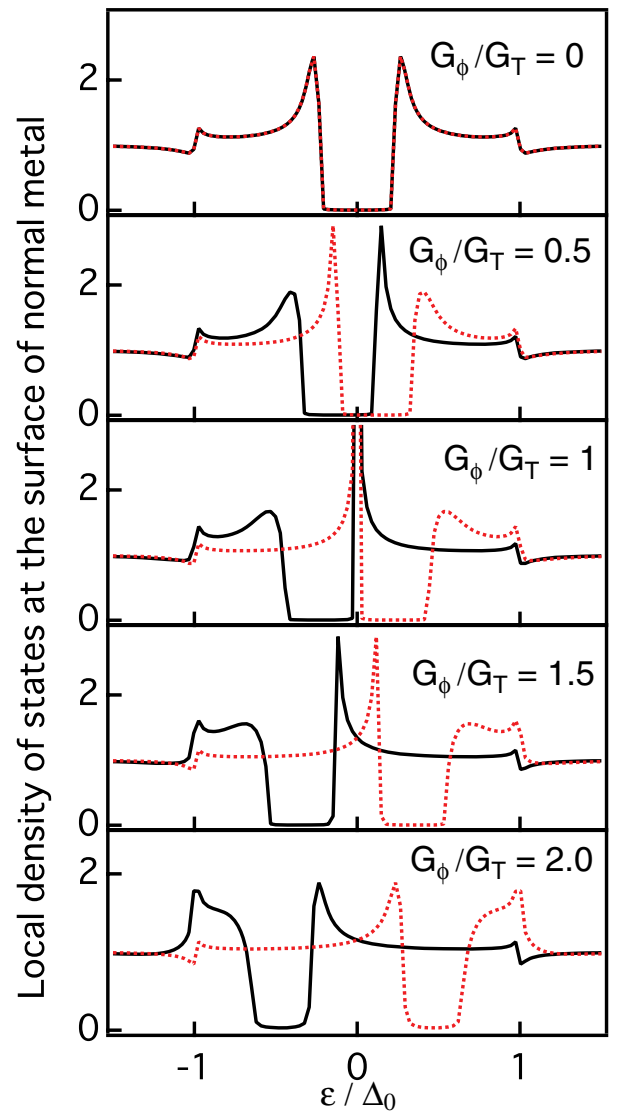

FIG. 2. (Color online) Local density of states at the surface of the normal metal with $R_{d} / R_{b}=0.2$. The results of $N(\epsilon,-L, v=1)$ and $N(\epsilon,-L, \nu=-1)$ are shown with the solid and broken lines, respectively.

\section{A. Density of states}

We first show the local density of states (LDOS) at a surface of the normal metal for several choices of $G_{\phi} / G_{T}$ in Fig. 2. Here, we choose the parameter $R_{d} / R_{b}=0.2$, which means the proximity effect is weak. The solid and broken lines are the results calculated for $v=1$ and -1 , respectively. At $G_{\phi} / G_{T}=0$, two LDOS for $v= \pm 1$ are identical to each other and show the minigap structure for $|\epsilon| \leqslant 0.2 \Delta_{0}$ due to the proximity effect. In Fig. 3, we show the pair spectral density defined in Eq. (22). At $G_{\phi} / G_{T}=0, K_{s}$ has a large positive peak around $\epsilon \approx 0.2 \Delta_{0}$. Therefore, the local pair density in Eq. (24) becomes positive. This means the penetration of even-frequency pairs into the normal metal. When we introduce $G_{\phi} / G_{T}$ at 0.5 , LDOS for $v=1$ shifts to negative direction, whereas that for $v=-1$ moves to positive direction. Correspondingly, large positive peaks in $K_{s}$ are separated into two as shown in Fig. 3. At $G_{\phi} / G_{T}=1.0$, two peaks in LDOS overlap each other. In the $K_{s}$ function, the large positive peak for $v=1$ totally cancels the large negative peak for $v=-1$. As discussed in a previous paper, ${ }^{7} G_{\phi} / G_{T}=1.0$ is a critical value. For $G_{\phi} / G_{T}<1.0$, the even-frequency Cooper pairs are dominant in the normal metal. On the other hand, for $G_{\phi} / G_{T}>1.0$, the fraction of odd-frequency Cooper pair increases with increasing $G_{\phi} / G_{T}$. In particular at $\epsilon=0$, the frequency symmetry of Cooper pairs is purely odd. When we increase $G_{\phi} / G_{T}=1.5$ and 2.0, the minigaps in two subspaces 


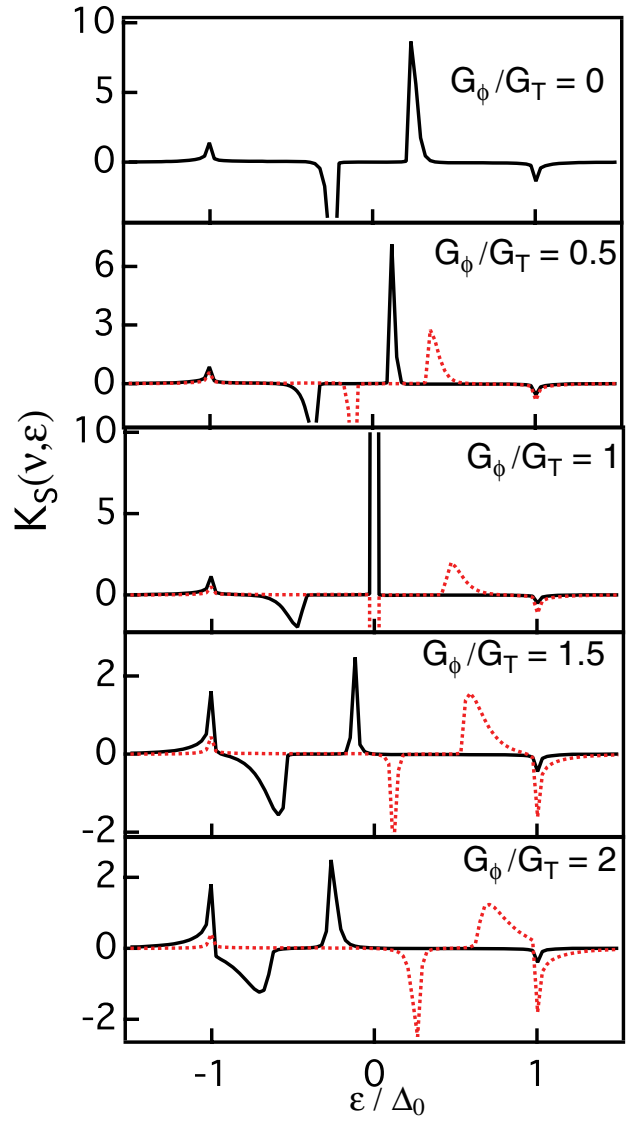

FIG. 3. (Color online) $K_{s}$ function at the surface of the normal metal. The local pair density is calculated from Eq. (24). The results of $K_{s}(\nu=1, \epsilon)$ and $K_{s}(v=-1, \epsilon)$ are shown with the solid and broken lines, respectively.

are separated from each other as shown in Fig. 2. At the same time, $K_{s}$ has a large negative peak in the low-energy region, which means the penetration of odd-frequency Cooper pairs into the normal metal.

\section{B. Impedance}

Next, we show the impedance as a function of temperature for several choices of $G_{\phi} / G_{T}$ in Fig. 4, where $\hbar \omega=0.1 \Delta_{0}$. We choose a boundary parameter as $R_{d} / R_{b}=0.2$ in Fig. 4 , which again means the proximity effect is weak. The results for $G_{\phi} / G_{T}=0$ in Fig. 4(a) show the typical and conventional behavior of impedance in NS junctions. The local impedance at the surface of normal metal $R_{L}$ and $X_{L}$ monotonically decreases with decreasing temperature far below $T_{C}$ and satisfies the robust relation $R_{L}<X_{L}$. The impedance of a NS bilayer $R_{\mathrm{NS}}$ and $X_{\mathrm{NS}}$ shows qualitatively similar behavior. Namely, the impedance satisfies $R_{\mathrm{NS}}<X_{\mathrm{NS}}$. These behaviors are a direct consequence of the fact that all Cooper pairs belong to even-frequency spin-singlet $s$-wave pairing symmetry. Such a characteristic feature remains even if we introduce $G_{\phi} / G_{T}$ by small amount up to 1.0 as shown in Fig. 4(b). In the presence of the spin-dependent potential at the NS interface, the odd-frequency spin-triplet $s$-wave Cooper pairs appear in the normal metal in addition to conventional even-frequency spin-singlet $s$-wave pairs. The fraction of odd-frequency

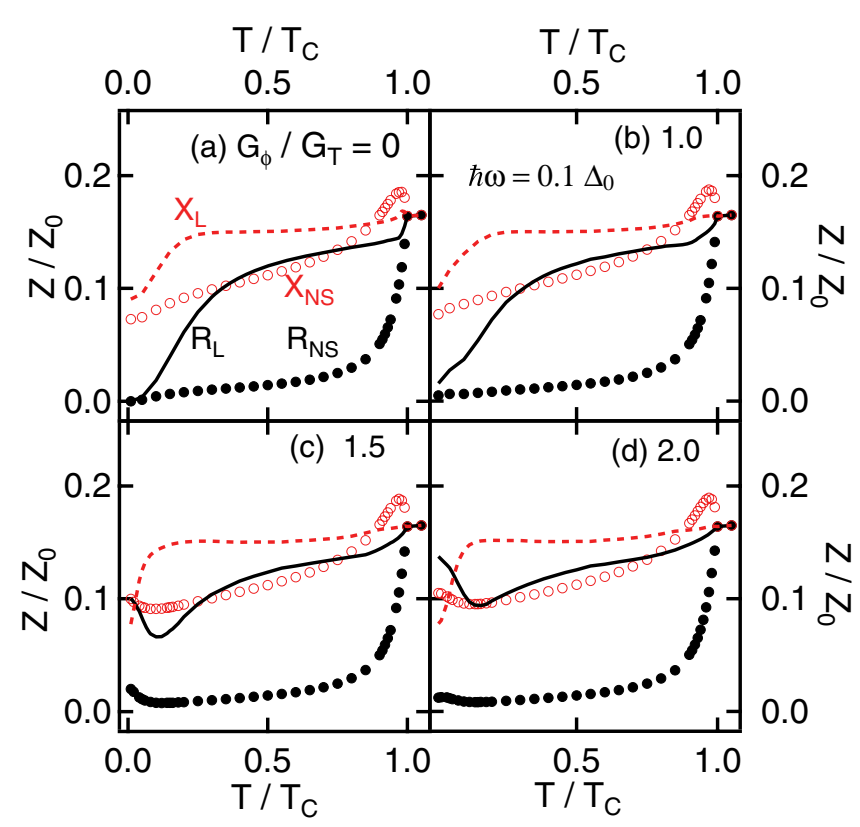

FIG. 4. (Color online) Impedance is plotted as a function of temperature for $R_{d} / R_{b}=0.2$ and $\hbar \omega=0.1 \Delta_{0}$. The length of the normal metal is fixed at $L / \xi_{T_{C}}=1$. The symbols $\left(R_{\mathrm{NS}}\right.$ and $\left.X_{\mathrm{NS}}\right)$ represent the results of impedance for the whole NS bilayer in Eq. (16). The lines $\left(R_{L}\right.$ and $\left.X_{L}\right)$ are the local impedance at the surface of the normal metal given in Eq. (15).

pairs is much smaller than that of even-frequency pairs for $G_{\phi} / G_{T} \leqslant 1$. However, $G_{\phi} / G_{T}$ exceeds unity as shown in Figs. 4(c) and 4(d), and the local impedance shows the unusual relation $R_{L}>X_{L}$ at low temperature for $T<T^{*}$, where $T^{*}$ is defined as the crossover temperature. In Figs. 4(c) and $4(\mathrm{~d}), T^{*}$ is about 0.02 for $G_{\phi} / G_{T}=1.5$ and is 0.06 for $G_{\phi} / G_{T}=2$, respectively. At the same time, $R_{\mathrm{NS}}$ and $X_{\mathrm{NS}}$ show the nonmonotonic dependence of temperature for $T<T^{*}$. For $G_{\phi} / G_{T} \geqslant 1$, the fraction of the odd-frequency Cooper pairs becomes larger than that of the even-frequency pairs. Thus, the anomalous behavior of impedance in Figs. 4(c) and $4(\mathrm{~d})$ is the direct evidence of the odd-frequency Cooper pairs in the normal metal. ${ }^{23}$

Such anomalous behavior of impedance $\left(R_{L}>X_{L}\right)$ is expected in a much wider temperature range when we consider stronger proximity effect. The results are shown in Figs. 5 and 6, where we choose $R_{d} / R_{b}=1$ in Fig. 5 and $R_{d} / R_{b}=5$
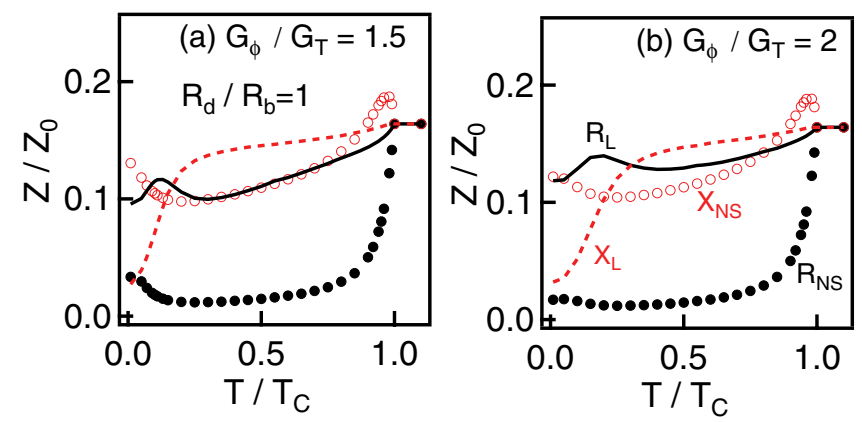

FIG. 5. (Color online) The results of impedance for $R_{d} / R_{b}=1$ and $\hbar \omega=0.1 \Delta_{0}$. 

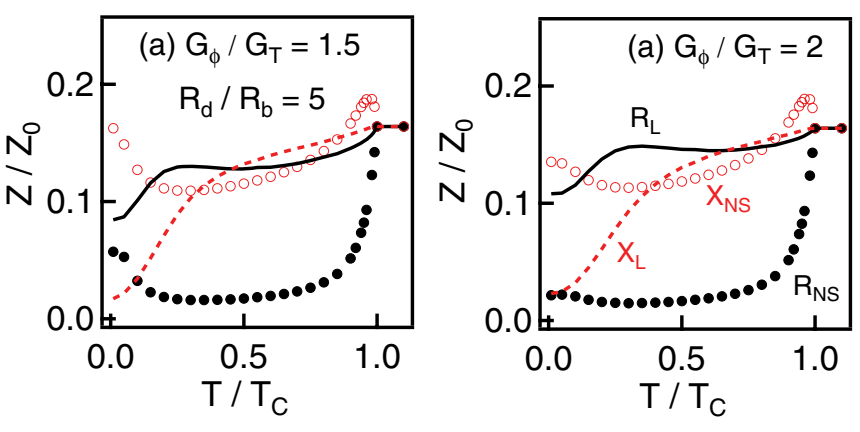

FIG. 6. (Color online) The results of impedance for $R_{d} / R_{b}=5$ and $\hbar \omega=0.1 \Delta_{0}$.

in Fig. 6. The frequency of electromagnetic field remains unchanged from $\hbar \omega=0.1 \Delta_{0}$ in both figures. At $G_{\phi} / G_{T}=$ 1.5 , for instance, the crossover temperature is $T^{*}=0.02 T_{C}$ for $R_{d} / R_{b}=0.2$ in Fig. $4(\mathrm{c}), T^{*}=0.17 T_{C}$ for $R_{d} / R_{b}=1$ in Fig. 5(a), and $T^{*}=0.5 T_{C}$ for $R_{d} / R_{b}=5$ in Fig. 6(a). In the same way, at $G_{\phi} / G_{T}=2.0, T^{*} / T_{C}$ is $0.06,0.3$, and 0.8 in Figs. 4(d), 5(b), and 6(b), respectively. Thus, we conclude that the anomalous relation in the local impedance $R_{L}>X_{L}$ can be observed in a wider temperature range for larger $R_{d} / R_{b}$. On the other hand, the impedance of the whole NS bilayer always shows the usual relation $R_{\mathrm{NS}}<X_{\mathrm{NS}}$. The even-frequency Cooper pairs in the superconductor dominate the impedance of the bilayer. The nonmonotonic temperature dependence of $R_{\mathrm{NS}}$ and $X_{\mathrm{NS}}$, however, can be seen for $T<T^{*}$.

Finally, we look into the impedance for several choices of the frequency of electromagnetic field in Fig. 7, where we choose $G_{\phi} / G_{T}=1.5$ and $R_{d} / R_{b}=5$. The frequency of electromagnetic field is chosen as $\hbar \omega / \Delta_{0}=0.01$ and 0.5 in Figs. 7(a) and 7(b), respectively. Here, we focus only on the local impedance $Z_{L}$. These results should be compared with Fig. 6(a) for $\hbar \omega / \Delta_{0}=0.1$. The crossover temperature to the anomalous relation is higher for smaller frequency. In Fig. 7(a), we find $T^{*} \sim 0.8 T_{C}$. Thus, it is easier to detect the anomalous relation $R>X$ in lower frequency in experiments. On the other hand, any sign for the odd-frequency pairs can not be seen in the results for high frequency at $\hbar \omega=0.5 \Delta_{0}$ in Fig. 7(b). Thus, we need to tune the frequency of electromagnetic field to be much smaller than $\Delta_{0} / \hbar$.

On the basis of the calculated results, we predict that the anomalous relation of the impedance $R>X$ due to the
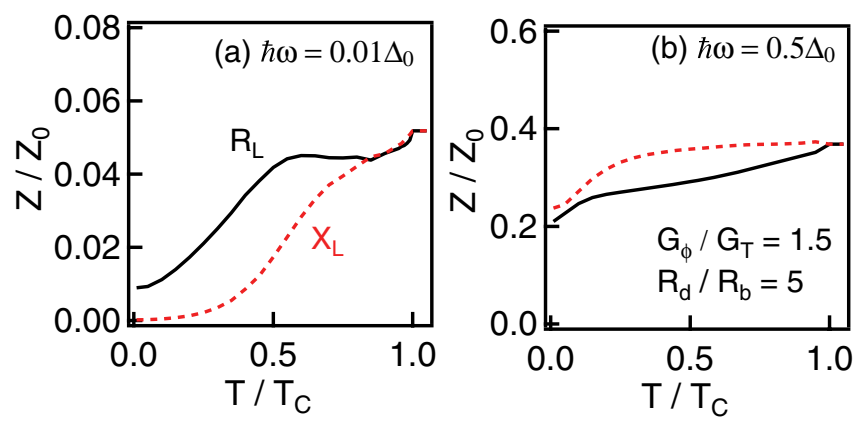

FIG. 7. (Color online) The results of local impedance for $R_{d} / R_{b}=5$ and $G_{\phi} / G_{T}=1.5$. We choose $\hbar \omega / \Delta_{0}=0.01$ in (a) and 0.5 in (b). These results should be compared with the results for $\hbar \omega / \Delta_{0}=0.1$ in Fig. 6(a). odd-frequency Cooper pairs would be observed for high value of $G_{\phi} / G_{T}$ and sufficiently low frequency of electromagnetic filed. The fabrication of NS bilayers using a thin ferromagnetic insulator $^{28}$ would realize large enough value of $G_{\phi} / G_{T}$. It is also important to note that, as shown in Ref. 34, in the case of thin ferromagnetic (F) film, the effects of the exchange field and the $G_{\phi} / G_{T}$ are equivalent. Therefore, the predicted anomalous behavior of impedance can be also realized in $\mathrm{S} / \mathrm{F}$ junctions with thin $\mathrm{F}$ layer. At the same time, the local impedance measurement is possible now. ${ }^{36}$ Thus, the conclusion of this paper could be confirmed in experiments.

\section{CONCLUSION}

We have studied the surface impedance $(Z=R-i X)$ of a normal-metal/superconductor bilayer, which has the spindependent potential at its junction interface. The complex conductivity is calculated from the quasiclassical Green's function which is obtained by solving the Usadel equation numerically. The effects of the spin-dependent potential at the interface are considered through the $G_{\phi}$ term in the Kupriyanov-Luckicev boundary condition at the junction interface. The spin-dependent potential produces the oddfrequency Cooper pairs in the normal metal. We conclude that the local impedance in the normal metal shows the unusual relationship $R>X$ when the odd-frequency Cooper pairs become dominant in the normal metal. The predicted results can be observed by recently developed local impedance measurement technique. In this paper, we consider the spinsinglet $s$-wave superconductor as a bulk superconductor. It is a challenging issue to extend this calculation available for unconventional superconductor, spin-singlet $d$ wave, ${ }^{38}$ spin-triplet $p$ wave, ${ }^{39}$ and topological superconductors. ${ }^{40}$ In these systems, it is known that the Andreev bound state or Majorana fermion governs charge transport. ${ }^{41}$ Indeed, we have shown a strong relationship between the Majorana fermions and the odd-frequency Cooper pairs. ${ }^{42}$

\section{ACKNOWLEDGMENT}

This work was supported by KAKENHI (Grant No. 22540355) and the 'Topological Quantum Phenomena' (Grant No. 22103002) Grant-in Aid for Scientific Research on Innovative Areas from the Ministry of Education, Culture, Sports, Science and Technology (MEXT) of Japan.

\section{APPENDIX: DERIVATION OF THE LOCAL CONDUCTIVITY}

The formula of the local conductivity was originally derived in Ref. 37. Here, we show an alternative derivation based on the quasiclassical Green's function method. ${ }^{43}$ The Usadel equation of spin-singlet $s$-wave superconducting junctions under the exchange potential reads as

$$
\begin{array}{r}
i \hbar \partial_{t_{1}} \check{T}_{3} \check{G}_{12}^{X}+i \hbar \partial_{t_{2}} \check{G}_{12}^{X} \check{T}_{3}-i \hbar D \vec{\partial} \check{I}^{X}+\left[-\check{V}+\check{\Delta}, \check{G}_{12}^{X}\right]=0, \\
\check{I}^{X}=\left\{\begin{array}{cl}
\int d t_{3} \check{G}_{13}^{X} \vec{\partial} \check{G}_{32}^{X} & \text { for } X=R, A, \\
\int d t_{3} \check{G}_{13}^{R} \vec{\partial} \check{G}_{32}^{K}+\check{G}_{13}^{K} \vec{\partial} \check{G}_{32}^{A} & \text { for } X=K,
\end{array}\right.
\end{array}
$$




$$
\begin{aligned}
& \check{V}=\left[\begin{array}{cc}
\boldsymbol{V}(\boldsymbol{r}) \cdot \boldsymbol{\sigma} & 0 \\
0 & \boldsymbol{V}(\boldsymbol{r}) \cdot \boldsymbol{\sigma}^{*}
\end{array}\right], \quad \check{\Delta}=\left[\begin{array}{cc}
0 & \Delta i \hat{\sigma}_{2} \\
\Delta^{*} i \hat{\sigma}_{2} & 0
\end{array}\right], \\
& \check{T}_{3}=\left[\begin{array}{cc}
\hat{1} & 0 \\
0 & -\hat{1}
\end{array}\right], \quad \check{G}_{12}^{X}=\left[\begin{array}{ll}
\hat{g}^{X} & \hat{f}^{X} \\
\hat{h}^{X} & \hat{k}^{X}
\end{array}\right]_{\left(\boldsymbol{r}, t_{1}, t_{2}\right)} \\
& \vec{\partial} \check{Y}_{i j}=\nabla_{\boldsymbol{r}} \check{Y}_{i j}-\frac{i e}{\hbar c} \boldsymbol{A}\left(\boldsymbol{r}, t_{i}\right)\left[\check{T}_{3}, \check{Y}_{i j}\right], \quad \check{Y}_{i j}=\check{Y}\left(\boldsymbol{r}, t_{i}, t_{j}\right)
\end{aligned}
$$

where $\hat{\sigma}_{j}$ for $j=1-3$ are the Pauli matrices, $\hat{1}$ is the unit matrix in spin space, and $\hat{.}$. means $2 \times 2$ matrices in spin space. The vector potential of electromagnetic field is given by $\boldsymbol{A}(\boldsymbol{r}, t)$. The Green's function $\breve{G}^{X}$ with $X=R, A$ and $K$ mean the retarded, the advanced and the Keldysh functions, which are represented in $4 \times 4$ matrix form indicated by.. . The electric current is given by

$$
\begin{aligned}
\boldsymbol{J}(\boldsymbol{r}, t)= & \frac{\pi \sigma_{0}}{8 e} \lim _{t^{\prime} \rightarrow t} \operatorname{Tr} \int d t_{1} \check{T}_{3}\left[\breve{G}^{R}\left(\boldsymbol{r}, t, t_{1}\right) \vec{\partial} \breve{G}^{K}\left(\boldsymbol{r}, t_{1}, t^{\prime}\right)\right. \\
& \left.+\check{G}^{K}\left(\boldsymbol{r}, t, t_{1}\right) \vec{\partial} \check{G}^{A}\left(\boldsymbol{r}, t_{1}, t^{\prime}\right)\right] .
\end{aligned}
$$

In the linear response regime with $\boldsymbol{A}(\boldsymbol{r}, t)=\boldsymbol{A}_{\omega} e^{-i \omega t}$, the current is expressed by

$$
\begin{gathered}
\boldsymbol{J}(\boldsymbol{r}, t)=\int \frac{d \omega}{2 \pi} \boldsymbol{J}(\boldsymbol{r}, \omega) e^{-i \omega t}, \\
\boldsymbol{J}(\boldsymbol{r}, \omega)=-i \frac{\pi \sigma_{0}}{8 \hbar c} \boldsymbol{A}_{\omega} \int \frac{d \epsilon}{2 \pi} \operatorname{Tr}\left[\check{T}_{3} \check{G}^{R}(\boldsymbol{r}, \epsilon+\hbar \omega) \check{T}_{3} \check{G}^{K}(\boldsymbol{r}, \epsilon)\right. \\
\left.+\check{T}_{3} \check{G}^{K}(\boldsymbol{r}, \epsilon+\hbar \omega) \check{T}_{3} \check{G}^{A}(\boldsymbol{r}, \epsilon)\right] .
\end{gathered}
$$

The Green's functions in this expression are calculated at $\boldsymbol{A}=$ 0 in equilibrium. The retarded function obeys

$$
\begin{aligned}
- & i \hbar D \nabla_{\boldsymbol{r}}\left\{\check{G}^{R}(\boldsymbol{r}, \epsilon) \nabla_{\boldsymbol{r}} \check{G}^{R}(\boldsymbol{r}, \epsilon)\right\} \\
& +\left[\epsilon \check{T}_{3}-\check{V}+\check{\Delta}, \check{G}^{R}(\boldsymbol{r}, \epsilon)\right]=0 .
\end{aligned}
$$

The advanced function and the Keldysh one are calculated from the relations

$$
\begin{aligned}
& \check{G}^{K}(\boldsymbol{r}, \epsilon)=\tanh \left(\frac{\epsilon}{2 k_{B} T}\right)\left[\check{G}^{R}(\boldsymbol{r}, \epsilon)-\check{G}^{A}(\boldsymbol{r}, \epsilon)\right], \\
& \check{G}^{A}(\boldsymbol{r}, \epsilon)=-\check{T}_{3}\left[\check{G}^{A}(\boldsymbol{r}, \epsilon)\right]^{\dagger} \check{T}_{3} .
\end{aligned}
$$

From the symmetry of the Usadel equation in Eq. (A7), we also find the relations

$\hat{k}^{R}(\boldsymbol{r}, \epsilon)=-\hat{\sigma}_{2} \hat{g}^{R}(\boldsymbol{r}, \epsilon) \hat{\sigma}_{2}, \quad \hat{h}^{R}(\boldsymbol{r}, \epsilon)=\hat{\sigma}_{2} \hat{f}^{R}(\boldsymbol{r}, \epsilon) \hat{\sigma}_{2}$.

Using relation $\boldsymbol{A}_{\omega}=-i(c / \omega) \boldsymbol{E}_{\omega}$, the current is represented as

$$
\begin{aligned}
\boldsymbol{J}(\boldsymbol{r}, \omega)= & -\frac{\pi \sigma_{0}}{4 \hbar \omega} \boldsymbol{E}_{\omega} \int \frac{d \epsilon}{2 \pi}\left[\operatorname { T r } \left\{\hat{g}(2)\left(\hat{g}(1)+\hat{g}^{\dagger}(1)\right)\right.\right. \\
& \left.-\hat{f}(2)\left(\hat{f}(1)-\hat{f}^{\dagger}(1)\right)\right\} \tanh \left[\frac{\epsilon}{2 k_{B} T}\right] \\
& -\operatorname{Tr}\left\{\left(\hat{g}(2)+\hat{g}^{\dagger}(2)\right) \hat{g}^{\dagger}(1)+(\hat{f}(2)\}\right. \\
& \left.\left.-\hat{f}^{\dagger}(2)\right) \hat{f}^{\dagger}(1) \tanh \left[\frac{\epsilon+\hbar \omega}{2 k_{B} T}\right]\right], \\
\hat{g}(1)= & \hat{g}^{R}(\boldsymbol{r}, \epsilon), \quad \hat{g}(2)=\hat{g}^{R}(\boldsymbol{r}, \epsilon+\hbar \omega), \\
\hat{f}(1)= & \hat{f}^{R}(\boldsymbol{r}, \epsilon), \quad \hat{f}(2)=\hat{f}^{R}(\boldsymbol{r}, \epsilon+\hbar \omega) .
\end{aligned}
$$

Here, the current is expressed only by the retarded Green's function, which can be decomposed into

$$
\begin{aligned}
& \hat{g}^{R}(\boldsymbol{r}, \epsilon)=g_{0}(\boldsymbol{r}, \epsilon) \hat{1}+\vec{g}(\boldsymbol{r}, \epsilon) \cdot \hat{\boldsymbol{\sigma}}, \\
& \hat{f}^{R}(\boldsymbol{r}, \epsilon)=f_{0}(\boldsymbol{r}, \epsilon) \hat{1}+\vec{f}(\boldsymbol{r}, \epsilon) \cdot \hat{\boldsymbol{\sigma}} .
\end{aligned}
$$

By using these relations, the current becomes

$$
\begin{aligned}
\boldsymbol{J}(\boldsymbol{r}, \omega)= & \frac{\pi \sigma_{0}}{\hbar \omega} \boldsymbol{E}_{\omega} \int \frac{d \epsilon}{2 \pi}\left[K _ { 1 } \left\{\tanh \left(\frac{\epsilon+\hbar \omega}{2 k_{B} T}\right)\right.\right. \\
& \left.-\tanh \left(\frac{\epsilon}{2 k_{B} T}\right)\right\}+i\left\{K_{2} \tanh \left(\frac{\epsilon+\hbar \omega}{2 k_{B} T}\right)\right. \\
+ & \left.\left.K_{3} \tanh \left(\frac{\epsilon}{2 k_{B} T}\right)\right\}\right], \\
K_{1}(\boldsymbol{r}, \epsilon, \omega)= & \sum_{j=0}^{3}\left[\operatorname{Re}\left\{g_{j}(\boldsymbol{r}, \epsilon+\hbar \omega)\right\} \operatorname{Re}\left\{g_{j}(\boldsymbol{r}, \epsilon)\right\}\right. \\
& \left.+\operatorname{Im}\left\{f_{j}(\boldsymbol{r}, \epsilon+\hbar \omega)\right\} \operatorname{Im}\left\{f_{j}(\boldsymbol{r}, \epsilon)\right\}\right], \\
K_{2}(\boldsymbol{r}, \epsilon, \omega)= & \sum_{j=0}^{3}\left[\operatorname{Re}\left\{f_{j}(\boldsymbol{r}, \epsilon)\right\} \operatorname{Im}\left\{f_{j}(\boldsymbol{r}, \epsilon+\hbar \omega)\right\}\right. \\
& \left.-\operatorname{Im}\left\{g_{j}(\boldsymbol{r}, \epsilon)\right\} \operatorname{Re}\left\{g_{j}(\boldsymbol{r}, \epsilon+\hbar \omega)\right\}\right], \\
K_{3}(\boldsymbol{r}, \epsilon, \omega)= & \sum_{j=0}^{3}\left[\operatorname{Re}\left\{f_{j}(\boldsymbol{r}, \epsilon+\hbar \omega)\right\} \operatorname{Im}\left\{f_{j}(\boldsymbol{r}, \epsilon)\right\}\right. \\
& \left.-\operatorname{Im}\left\{g_{j}(\boldsymbol{r}, \epsilon+\hbar \omega)\right\} \operatorname{Re}\left\{g_{j}(\boldsymbol{r}, \epsilon)\right\}\right] .
\end{aligned}
$$

Since $\boldsymbol{J}(\boldsymbol{r}, \omega)=\left[\sigma_{1}(\boldsymbol{r}, \omega)+i \sigma_{2}(\boldsymbol{r}, \omega)\right] \boldsymbol{E}_{\omega}$, we obtain

$$
\begin{aligned}
\frac{\sigma_{1}(\boldsymbol{r}, \omega)}{\sigma_{0}}= & \int \frac{d \epsilon}{2 \hbar \omega} K_{1}(\boldsymbol{r}, \epsilon, \omega)\left[\tanh \left(\frac{\epsilon+\hbar \omega}{2 k_{B} T}\right)\right. \\
& \left.-\tanh \left(\frac{\epsilon}{2 k_{B} T}\right)\right], \\
\frac{\sigma_{2}(\boldsymbol{r}, \omega)}{\sigma_{0}}= & \int \frac{d \epsilon}{2 \hbar \omega}\left[K_{2}(\boldsymbol{r}, \epsilon, \omega) \tanh \left(\frac{\epsilon+\hbar \omega}{2 k_{B} T}\right)\right. \\
& \left.+K_{3}(\boldsymbol{r}, \epsilon, \omega) \tanh \left(\frac{\epsilon}{2 k_{B} T}\right)\right] .
\end{aligned}
$$

When the magnetic moment is $\boldsymbol{V}=V_{3} \boldsymbol{e}_{3}$ with $\boldsymbol{e}_{3}$ being the unit vector of the third axis in spin space, we find that $g_{1}=g_{2}=f_{1}=f_{2}=0$. By applying the parametrization

$\hat{g}^{R}(\boldsymbol{r}, \epsilon)=g_{0}(\boldsymbol{r}, \epsilon)+g_{3}(\boldsymbol{r}, \epsilon) \hat{\sigma}_{3}=\sum_{\nu= \pm 1} \cos \theta_{\nu}(\boldsymbol{r}, \epsilon) \frac{\hat{1}+v \hat{\sigma}_{3}}{2}$,

$$
\hat{f}^{R}(\boldsymbol{r}, \epsilon)=f_{0}(\boldsymbol{r}, \epsilon)+f_{3}(\boldsymbol{r}, \epsilon) \hat{\sigma}_{3}=\sum_{\nu= \pm 1} \sin \theta_{v}(\boldsymbol{r}, \epsilon) \frac{\hat{1}+\nu \hat{\sigma}_{3}}{2},
$$

the Usadel equation is decomposed into two equations

$$
-i \hbar D \nabla_{r}^{2} \theta_{v}+2\left(\epsilon+v V_{3}\right) \sin \theta_{v}-2 i \Delta \cos \theta_{v}=0,
$$

where $v= \pm 1$ corresponds to two Nambu spaces. This equation becomes Eq. (1) in a normal metal because of $V_{3}=\Delta=0$. The current is represented in terms 
of $\theta_{\nu}(\boldsymbol{r}, \epsilon)$ as

$$
\begin{aligned}
\boldsymbol{J}(\boldsymbol{r}, \omega)= & \frac{\pi \sigma_{0}}{2 \hbar \omega} \boldsymbol{E}_{\omega} \int \frac{d \epsilon}{2 \pi}\left[\overline { K } _ { 1 } \left\{\tanh \left(\frac{\epsilon+\hbar \omega}{2 k_{B} T}\right)\right.\right. \\
& \left.-\tanh \left(\frac{\epsilon}{2 k_{B} T}\right)\right\}+i\left\{\bar{K}_{2} \tanh \left(\frac{\epsilon+\hbar \omega}{2 k_{B} T}\right)\right. \\
& \left.\left.+\bar{K}_{3} \tanh \left(\frac{\epsilon}{2 k_{B} T}\right)\right\}\right], \\
\bar{K}_{1}(\boldsymbol{r}, \epsilon, \omega)= & \sum_{\nu= \pm 1}\left[\operatorname{Re}\left\{\cos \theta_{v}(\boldsymbol{r}, \epsilon)\right\} \operatorname{Re}\left\{\cos \theta_{\nu}(\boldsymbol{r}, \epsilon+\hbar \omega)\right\}\right. \\
& \left.+\operatorname{Im}\left\{\sin \theta_{\nu}(\boldsymbol{r}, \epsilon)\right\} \operatorname{Im}\left\{\sin \theta_{v}(\boldsymbol{r}, \epsilon+\hbar \omega)\right\}\right],
\end{aligned}
$$

$$
\begin{aligned}
\bar{K}_{2}(\boldsymbol{r}, \epsilon, \omega)= & \sum_{\nu= \pm 1}\left[\operatorname{Re}\left\{\sin \theta_{v}(\boldsymbol{r}, \epsilon)\right\} \operatorname{Im}\left\{\sin \theta_{\nu}(\boldsymbol{r}, \epsilon+\hbar \omega)\right\}\right. \\
& \left.-\operatorname{Im}\left\{\cos \theta_{v}(\boldsymbol{r}, \epsilon)\right\} \operatorname{Re}\left\{\cos \theta_{\nu}(\boldsymbol{r}, \epsilon+\hbar \omega)\right\}\right],
\end{aligned}
$$

$$
\begin{aligned}
\bar{K}_{3}(\boldsymbol{r}, \epsilon, \omega)= & \sum_{\nu= \pm 1}\left[\operatorname{Re}\left\{\sin \theta_{\nu}(\boldsymbol{r}, \epsilon+\hbar \omega)\right\} \operatorname{Im}\left\{\sin \theta_{\nu}(\boldsymbol{r}, \epsilon)\right\}\right. \\
& \left.-\operatorname{Im}\left\{\cos \theta_{\nu}(\boldsymbol{r}, \epsilon+\hbar \omega)\right\} \operatorname{Re}\left\{\cos \theta_{\nu}(\boldsymbol{r}, \epsilon)\right\}\right]
\end{aligned}
$$

We reach the expression of the local impedance in Eqs. (7) and (8).

\section{APPENDIX B: DERIVATION OF THE IMPEDANCE OF NS BILAYERS}

Let us consider that the electromagnetic field propagating the $+x$ direction is described by

$$
\boldsymbol{E}=E(x) \hat{\boldsymbol{y}} e^{-i \omega t}, \quad \boldsymbol{H}=H(x) \hat{z} e^{-i \omega t},
$$

where $\omega>0$ is a frequency of the electromagnetic field, $\hat{\boldsymbol{y}}(\hat{\boldsymbol{z}})$ is the unit vector in the $y(z)$ direction. In such a situation, the Maxwell's equation in a metal and a superconductor reads as

$$
-\partial_{x} H(x)=\frac{4 \pi \sigma}{c} E(x), \quad \partial_{x} E(x)=i \frac{\omega}{c} H(x),
$$

where $\sigma$ is the complex conductivity. By combining these equations, it is possible to obtain

$$
\left[\partial_{x}^{2}+i \frac{4 \pi \sigma \omega}{c^{2}}\right] H(x)=0, \quad\left[\partial_{x}^{2}+i \frac{4 \pi \sigma \omega}{c^{2}}\right] E(x)=0 .
$$

Therefore, the electromagnetic field in a metal is described by

$$
\begin{aligned}
H(x) & =H_{1} e^{i k x}+H_{2} e^{-i k x}, \quad E(x)=E_{1} e^{i k x}+E_{2} e^{-i k x}, \\
k^{2} & =i \frac{4 \pi \omega \sigma}{c^{2}} .
\end{aligned}
$$

When we consider a semi-infinite surface of a metal or a superconductor, the first equation in Eq. (B2) with a condition $H_{2}=E_{2}=0$ gives $-i k H_{1}=(4 \pi \sigma / c) E_{1}$. The surface impedance is defined by

$$
Z=\frac{4 \pi}{c} \frac{E_{1}}{H_{1}}=\frac{-i k}{\sigma}=-i \sqrt{\frac{4 \pi i \omega}{c^{2} \sigma}} .
$$

In a normal metal, the complex conductivity is expressed by $\sigma_{n}=\sigma_{0}(1+i \omega \tau) /\left\{1+(\omega \tau)^{2}\right\}$ with $\sigma_{0}=\left(n_{n} e^{2} \tau / m\right), \tau$, and $n_{n}$ being the Drude conductivity, the mean-free time due to elastic impurity scatterings, and the normal electron density. In the low-frequency limit $\omega \ll 1 / \tau$, we find $\sigma_{n} \approx \sigma_{0}$ and

$$
\begin{aligned}
& k_{n}=(1+i) / \delta_{\text {skin }}, \quad \delta_{\text {skin }}=\sqrt{\frac{c^{2}}{2 \pi \sigma_{0} \omega}}, \\
& Z_{n}=R_{n}-i X_{n}=(1-i) Z_{0}, \quad Z_{0}=\sqrt{\frac{2 \pi \omega}{c^{2} \sigma_{0}}} .
\end{aligned}
$$

For a superconductor, we also obtain in the limit of $\omega \ll \Delta_{0} \ll$ $1 / \tau$

$$
\begin{aligned}
& \sigma_{s}=\sigma_{0}+i \frac{n_{s} e^{2}}{m \omega}, \quad k_{s}=\frac{i}{\lambda_{L}}, \quad \frac{1}{\lambda_{L}^{2}}=\frac{4 \pi n_{s} e^{2}}{m c^{2}}, \\
& Z_{\mathrm{S}}=R_{\mathrm{S}}-i X_{\mathrm{S}} \approx-i Z_{1}, \quad Z_{1}=\sqrt{\frac{4 \pi \omega}{c^{2} \sigma_{0}} \frac{n_{n}}{n_{s}} \omega \tau .}
\end{aligned}
$$

Next, we derive the impedance of NS bilayers as shown in Fig. 1. The electromagnetic field is described as

$$
H(x)=H_{1} e^{i k_{\mathrm{N}} x}+H_{2} e^{-i k_{\mathrm{N}} x}, \quad E(x)=E_{1} e^{i k_{\mathrm{N}} x}+E_{2} e^{-i k_{\mathrm{N}} x}
$$

in the normal metal $(-L<x<0)$ and

$$
H(x)=H_{s} e^{i k_{s} x}, \quad E(x)=E_{s} e^{i k_{s} x}
$$

in the superconductor $(0<x)$. The complex conductivity in the normal metal $\sigma_{\mathrm{N}}$ may be modified by the proximity effect and is different from $\sigma_{0}$. The wave number in the normal metal in Eq. (B9) is given by $k_{\mathrm{N}}^{2}=4 \pi i \omega \sigma_{\mathrm{N}} / c^{2}$. We use the Maxwell's equation to have boundary conditions at the NS interface (i.e., $x=0$ ). From the first equation in Eq. (B2), we obtain

$$
\frac{1}{\sigma_{\mathrm{N}}}\left[k_{\mathrm{N}} H_{1}-i k_{\mathrm{N}} H_{2}\right]=\frac{k_{s} H_{s}}{\sigma_{s}} .
$$

Here, we impose the continuity of the electric field at $x=0$ (i.e., $E_{s}=E_{1}+E_{2}$ ). At the same time, we also assume the continuity of magnetic field $H_{1}+H_{2}=H_{s}$. This condition also corresponds to the continuity of $\partial_{x} E(x)$ through the Eq. (B2). From these conditions, we obtain the magnetic and electric field in a normal metal:

$$
H(x)=\frac{H_{s}}{2}\left[\left(1+\frac{Z_{\mathrm{S}}}{Z_{\mathrm{N}}}\right) e^{i k_{N} x}+\left(1-\frac{Z_{\mathrm{S}}}{Z_{\mathrm{N}}}\right) e^{-i k_{N} x}\right],
$$

$$
\begin{aligned}
& \frac{4 \pi \sigma_{N}}{c} E(x) \\
& \quad=-i k_{N} \frac{H_{s}}{2}\left[\left(1+\frac{Z_{\mathrm{S}}}{Z_{\mathrm{N}}}\right) e^{i k_{N} x}-\left(1-\frac{Z_{\mathrm{S}}}{Z_{\mathrm{N}}}\right) e^{-i k_{N} x}\right] .
\end{aligned}
$$

The impedance at a surface of the NS structure becomes

$$
Z_{\mathrm{NS}}=\frac{4 \pi}{c} \frac{E(-L)}{H(-L)}=Z_{N} \frac{Z_{\mathrm{S}} \cos k_{\mathrm{N}} L-i Z_{\mathrm{N}} \sin k_{\mathrm{N}} L}{Z_{\mathrm{N}} \cos k_{\mathrm{N}} L-i Z_{\mathrm{S}} \sin k_{\mathrm{N}} L},
$$

with $Z_{\mathrm{N}}=-i k_{\mathrm{N}} / \sigma_{\mathrm{N}}$. 
${ }^{1}$ V. L. Berezinskii, JETP Lett. 20, 287 (1974) [Pis'ma Zh. Eksp. Teor. Fiz. 20, 628 (1974)].

${ }^{2}$ F. S. Bergeret, A. F. Volkov, and K. B. Efetov, Phys. Rev. Lett. 86, 4096 (2001); Rev. Mod. Phys. 77, 1321 (2005).

${ }^{3}$ Y. Asano, Y. Tanaka, and A. A. Golubov, Phys. Rev. Lett. 98, 107002 (2007).

${ }^{4}$ V. Braude and Yu. V. Nazarov, Phys. Rev. Lett. 98, 077003 (2007).

${ }^{5}$ M. Eschrig and T. Löfwander, Nat. Phys. 4, 138 (2008).

${ }^{6}$ Ya. V. Fominov, A. F. Volkov, and K. B. Efetov, Phys. Rev. B 75, 104509 (2007).

${ }^{7}$ J. Linder, T. Yokoyama, and A. Sudbo, Phys. Rev. B 77, 174507 (2008); J. Linder, T. Yokoyama, Y. Tanaka, Y. Asano, and A. Sudbo, ibid. 77, 174505 (2008); J. Linder, T. Yokoyama, A. Sudbø, and M. Eschrig, Phys. Rev. Lett. 102, 107008 (2009).

${ }^{8}$ T. Yokoyama, Y. Tanaka, and A. A. Golubov, Phys. Rev. B 72, 052512 (2005); 73, 094501 (2006).

${ }^{9}$ T. Yokoyama, Y. Tanaka, and A. A. Golubov, Phys. Rev. B 75, 094514 (2007); Y. Sawa, T. Yokoyama, Y. Tanaka, and A. A. Golubov, ibid. 75, 134508 (2007).

${ }^{10}$ K. Halterman, P. H. Barsic, and O. T. Valls, Phys. Rev. Lett. 99, 127002 (2007); P. H. Barsic and O. T. Valls, Phys. Rev. B 79, 014502 (2009).

${ }^{11}$ M. Houzet and A. I. Buzdin, Phys. Rev. B 76, 060504(R) (2007).

${ }^{12}$ R. S. Keizer, S. T. B. Goennenwein, T. M. Klapwijk, G. Miao, G. Xiao, and A. Gupta, Nature (London) 439, 825 (2006).

${ }^{13}$ M. S. Anwar, F. Czeschka, M. Hesselberth, M. Porcu, and J. Aarts, Phys. Rev. B 82, 100501(R) (2010).

${ }^{14}$ T. S. Khaire, M. A. Khasawneh, W. P. Pratt Jr., and N. O. Birge, Phys. Rev. Lett. 104, 137002 (2010).

${ }^{15}$ J. W. A. Robinson, J. D. S. Witt, and M. G. Blamire, Science 329, 59 (2010).

${ }^{16}$ Y. Tanaka, A. A. Golubov, S. Kashiwaya, and M. Ueda, Phys. Rev. Lett. 99, 037005 (2007); Y. Tanaka, Y. Tanuma, and A. A. Golubov, Phys. Rev. B 76, 054522 (2007).

${ }^{17}$ Y. Tanaka and A. A. Golubov, Phys. Rev. Lett. 98, 037003 (2007).

${ }^{18}$ Y. Tanaka and S. Kashiwaya, Phys. Rev. B 70, 012507 (2004); Y. Tanaka, S. Kashiwaya, and T. Yokoyama, ibid. 71, 094513 (2005).

${ }^{19}$ Y. Tanaka, Y. Asano, A. A. Golubov, and S. Kashiwaya, Phys. Rev. B 72, 140503(R) (2005).

${ }^{20}$ Y. Asano, Y. Tanaka, and S. Kashiwaya, Phys. Rev. Lett. 96, 097007 (2006).

${ }^{21}$ Y. Asano, Y. Tanaka, A. A. Golubov, and S. Kashiwaya, Phys. Rev. Lett. 99, 067005 (2007).

${ }^{22}$ Ya. V. Fominov, JETP Lett. 86, 732 (2007) [Pis'ma Zh. Eksp. Teor. Fiz. 86, 842 (2007)].

${ }^{23}$ Y. Asano, A. A. Golubov, Ya. V. Fominov, and Y. Tanaka, Phys. Rev. Lett. 107, 087001 (2011).

${ }^{24}$ D. C. Mattis and J. Bardeen, Phys. Rev. 111, 412 (1958).
${ }^{25}$ S. B. Nam, Phys. Rev. 156, 470 (1967).

${ }^{26}$ A. A. Abrikosov, L. P. Gorkov, and I. E. Dzyaloshinski, Methods of Quantum Field Theory in Statistical Physics (Dover, New York, 1975). The negative pair density, discussed in this paper, implies that the corresponding coefficient describing the response of the current to the vector potential becomes negative in equations of the standard theory of superconductivity.

${ }^{27}$ Y. Maeno, H. Hashimoto, K. Yoshida, S. Nishizaki, T. Fujita, J. G. Bednorz, and F. Lichtenberg, Nature (London) 372, 532 (1994).

${ }^{28}$ K. Senapati, M. G. Blamire, and Z. H. Barber, Nat. Mater. 10, 849 (2011).

${ }^{29}$ K. D. Usadel, Phys. Rev. Lett. 25, 507 (1970).

${ }^{30}$ Y. Tanaka and S. Kashiwaya, J. Phys. Soc. Jpn. 69, 1152 (2000); S. Kawabata, S. Kashiwaya, Y. Asano, Y. Tanaka, and A. A. Golubov, Phys. Rev. B 74, 180502(R) (2006); S. Kawabata, Y. Asano, Y. Tanaka, A. A. Golubov, and S. Kashiwaya, Phys. Rev. Lett. 104, 117002 (2010).

${ }^{31}$ M. Yu. Kupriyanov and V. F. Lukichev, Sov. Phys.-JETP 67, 1163 (1988) [Zh. Eksp. Teor. Fiz. 94, 139 (1988)].

${ }^{32}$ D. Huertas-Hernando, Yu. V. Nazarov, and W. Belzig, Phys. Rev. Lett. 88, 047003 (2002).

${ }^{33}$ A. Cottet, Phys. Rev. B 76, 224505 (2007).

${ }^{34}$ D. Yoshizaki, A. A. Golubov, Y. Tanaka, and Y. Asano, Jpn. J. Appl. Phys. 51, 010108 (2012).

${ }^{35}$ T. Yokoyama, Y. Tanaka, and N. Nagaosa, Phys. Rev. Lett. 106, 246601 (2011).

${ }^{36}$ T. Machida, M. B. Gaifullin, S. Ooi, T. Kato, H. Sakata, and K. Hirata, Jpn. J. Appl. Phys. 49, 116701 (2010); Appl. Phys. Express 2, 025006 (2009).

${ }^{37}$ Ya. V. Fominov, M. Houzet, and L. I. Glazman, Phys. Rev. B 84, 224517 (2011).

${ }^{38}$ Y. Tanaka and S. Kashiwaya, Phys. Rev. Lett. 74, 3451 (1995); Y. Asano, Y. Tanaka, and S. Kashiwaya, Phys. Rev. B 69, 134501 (2004); Y. Tanaka, Y. V. Nazarov, and S. Kashiwaya, Phys. Rev. Lett. 90, 167003 (2003); S. Kashiwayaa, Y. Tanakab, N. Teradaa, M. Koyanagia, S. Uenoa, L. Alff, H. Takashimaa, Y. Tanumab, and K. Kajimura, J. Phys. Chem. Solids 59, 10 (1998).

${ }^{39}$ M. Yamashiro, Y. Tanaka, and S. Kashiwaya, Phys. Rev. B 56, 7847 (1997); M. Yamashiro, Y. Tanaka, Y. Tanuma, and S. Kashiwaya, J. Phys. Soc. Jpn. 67, 3224 (1998); S. Kashiwaya, H. Kashiwaya, H. Kambara, T. Furuta, H. Yaguchi, Y. Tanaka, and Y. Maeno, Phys. Rev. Lett. 107, 077003 (2011).

${ }^{40}$ M. Sato and S. Fujimoto, Phys. Rev. B 79, 094504 (2009); Y. Tanaka, T. Yokoyama, A. V. Balatsky, and N. Nagaosa, ibid. 79, 060505 (2009); J. D. Sau, R. M. Lutchyn, S. Tewari, and S. Das Sarma, Phys. Rev. Lett. 104, 040502 (2010); J. Alicea, Phys. Rev. B 81, 125318 (2010).

${ }^{41}$ S. Kashiwaya and Y. Tanaka, Rep. Prog. Phys. 63, 1641 (2000); Y. Tanaka, M. Sato, and N. Nagaosa, J. Phys. Soc. Jpn. 81, 011013 (2012).

${ }^{42}$ Y. Asano and Y. Tanaka, arXiv:1204.4226.

${ }^{43}$ Ya. V. Fominov (private communication). 